CERN-TH/2001-127

hep-lat/0105010

\title{
Quenched Finite Volume Logarithms
}

\author{
P.H. DAMGAARD* \\ Theory Division \\ CERN \\ CH-1211 Geneva 23 \\ Switzerland
}

October 29, 2018

\begin{abstract}
Quenched chiral perturbation theory is used to compute the first finite volume correction to the chiral condensate. The correction diverges logarithmically with the four-volume $V$. We point out that with dynamical quarks one can obtain both the chiral condensate and the pion decay constant from the distributions of the lowest Dirac operator eigenvalues.
\end{abstract}

\footnotetext{
*On leave from: The Niels Bohr Institute, Blegdamsvej 17, DK-2100 Copenhagen, Denmark.
} 


\section{Introduction}

Finite-size effects in lattice gauge theory are usually considered undesireable features of numerical simulations. There are exceptions: near critical points, for instance, finite-size scaling applies the Renormalization Group in finite volumes to extract critical indices of the infinite volume system. Also away from critical points one can use knowledge of the finite volume behavior to learn about the infinite volume theory. When this is the case, one is turning the apparent disadvantage of being restricted to small volumes into a distinct advantage.

Ordinarily finite-volume computations in gauge theories still involve the full complexity of such strongly coupled theories. A program to overcome this obstacle was initiated several years ago by Gasser and Leutwyler [1, 2]. Let the finite (euclidean) 4-volume be of size $V=L_{x} L_{y} L_{z} L_{t}\left(L_{t}=1 / T\right)$. The idea is that for $L_{i} \gg 1 / \Lambda_{Q C D}$ the euclidean partition function (or appropriate generating functional) is dominated by the lowest-mass excitations. If chiral symmetry is spontaneously broken in the infinite volume limit these light particles are the pseudo-Goldstone bosons, whose interactions vanish at zero momentum transfer. The theory governing these excitations is a chiral Lagrangian. Although non-renormalizable, this theory can be treated perturbatively in the sense of an effective Lagrangian. Combined with the description of the fundamental degrees of freedom in the underlying gauge theories, this allows for precise finite-volume computations in these otherwise strongly coupled theories.

There are two finite-volume regimes to consider. The first is the one connected with the usual infinite volume limit of the theory. If $M$ denotes the mass of the pseudo-Goldstone boson, one requires $1 / M \ll$ $L_{i}$ so that the particle "fits well inside the box", and the finite volume $V$ is a minor perturbation. In this regime usual chiral perturbation theory is applicable. The other regime is $1 / M \gg L_{i}$ : if the volume is much larger than the scale set by the gauge theory, $L_{i} \gg 1 / \Lambda_{Q C D}$, the euclidean partition function is still dominated by the lightest excitations, but ordinary chiral perturbation theory fails. In this region a different expansion must be applied [2]. This paper concerns (partially) quenched theory in the latter regime. We shall also comment on how a known result from the unquenched theory can be used to extract the pion decay constant $F$ in an unusual, but quite simple way.

It is worthwhile stressing that although the finite volumes which are being probed here are unphysical in the sense that the pion correlation length exceeds the boundaries of the finite volume, the observables one can extract in this way are infinite-volume quantities. It is precisely the purpose to use unphysical finite-volume expectation values to extract these infinite-volume quantities directly, without any numerical extrapolation to the infinite-volume limit.

\section{The Chiral Condensate}

We begin with a brief review of the Feynman rules for partially quenched chiral perturbation theory based on the replica method [3]. Denoting the number of ordinary physical ("sea") quarks by $N_{f}$, one adds (in its simplest form) $N_{v}$ "valence" quarks of common mass $m_{v}$ to theory, and takes the limit $N_{v} \rightarrow 0$ in the end. The starting point is then an effective theory based on the coset of

$$
\mathrm{U}\left(N_{f}+N_{v}\right) \times \mathrm{U}\left(N_{f}+N_{v}\right) \rightarrow \mathrm{U}\left(N_{f}+N_{v}\right)
$$

including the flavor singlet field. The Lagrangian can be parametrized

$$
\mathcal{L}=\frac{F^{2}}{4} \operatorname{Tr}\left(\partial_{\mu} U(x) \partial^{\mu} U^{\dagger}(x)\right)-\frac{\Sigma}{2} \operatorname{Tr} \mathcal{M}\left(U(x)+U^{\dagger}(x)\right)+\frac{m_{0}^{2}}{2 N_{c}} \Phi_{0}^{2}(x)+\frac{\alpha}{2 N_{c}} \partial_{\mu} \Phi_{0}(x) \partial^{\mu} \Phi_{0}(x) .
$$


when keeping only the usual leading terms in the small momentum expansion. The field $U(x) \equiv$ $\exp [i \sqrt{2} \Phi(x) / F] \in \mathrm{U}\left(N_{f}+N_{v}\right)$ lives on the Goldstone manifold, and we have separated out the flavor singlet combination $\Phi_{0}(x) \equiv \operatorname{Tr} \Phi(x)$. For this reason it is convenient to work in the quark basis 断 where $\Phi_{i j} \sim \bar{\psi}_{i} \psi_{j}$. Let $M_{i j}^{2} \equiv\left(m_{i}+m_{j}\right) \Sigma / F^{2}$, and

$$
\mathcal{F}\left(p^{2}\right) \equiv 1+\frac{m_{0}^{2}+\alpha p^{2}}{N_{c}}\left(\frac{N_{v}}{p^{2}+M_{v v}^{2}}+\sum_{f=1}^{N_{f}} \frac{1}{p^{2}+M_{f f}^{2}}\right) .
$$

The momentum-space propagator for the off-diagonal mesons $\Phi_{i j} \sim \bar{\psi}_{i} \psi_{j}, i \neq j$ is then read off to be

$$
D_{i j}\left(p^{2}\right)=\frac{1}{p^{2}+M_{i j}^{2}}
$$

while the corresponding propagator for the diagonal combination $\Phi_{i i} \sim \bar{\psi}_{i} \psi_{i}$ is

$$
G_{i j}\left(p^{2}\right)=\frac{\delta_{i j}}{\left(p^{2}+M_{i i}^{2}\right)}-\frac{\left(m_{0}^{2}+\alpha p^{2}\right) / N_{c}}{\left(p^{2}+M_{i i}^{2}\right)\left(p^{2}+M_{j j}^{2}\right) \mathcal{F}\left(p^{2}\right)} .
$$

With these rules it is straightforward to take the partially quenched limit of chiral perturbation theory: one simply sends $N_{v} \rightarrow 0$ at the end of the calculation. It is simple to show [3] that it is equivalent to partially quenched chiral perturbation theory based on the supersymmetric formulation [4, 5, 6], but we find the replica method easier to use. It is straightforward to extend the method to a more elaborate chiral Lagrangian involving higher order terms in the momentum expansion. Here we restrict ourselves to the $\mathcal{O}\left(p^{2}\right)$ Lagrangian, supplemented with the lowest order $\Phi_{0}$-terms (1).

The conventional regime in which to apply (partially quenched) chiral perturbation is the one where the four-volume $V$ well encloses the pseudo-Goldstone bosons of masses $M_{i j}$, i.e., $L \gg 1 / M_{i j}$. Although the four-volume $V$ then can be kept finite, the correlation length does not reach from boundary to boundary, and the theory looks like a mildly perturbed infinite-volume theory. In this regime chiral perturbation theory can be arranged as a systematic expansion, roughly speaking in the small momentum $p$. This is clearly an obvious regime of interest for lattice gauge theory.

Actually, as pointed out first by Gasser and Leutwyler [1], another finite-volume regime can also be used to extract physical observables. This is the extreme finite-volume regime where $L \ll 1 / M_{i j}$. Although the pseudo-Goldstone bosons here apparently violate the bound which one would normally impose on the finite-size system, one can still perform analytical calculations here, and thus extract physical quantities by comparison with Monte Carlo data. This regime is governed by a perturbative theory of the chiral Lagrangian which is sometimes called the $\epsilon$-expansion [2] (not to be confused with an expansion away from critical dimensions). Let us first introduce the mean length scale

$$
L \equiv V^{1 / 4} \text {. }
$$

The counting of orders of $\epsilon$ is defined by fixing $1 / L=\mathcal{O}(\epsilon)$. The non-zero momentum modes thus count as $p \sim \mathcal{O}(\epsilon)$ too, while $m_{i} \sim \mathcal{O}\left(\epsilon^{4}\right)$ (and hence $M_{i j} \sim \mathcal{O}\left(\epsilon^{2}\right) ; \mu_{i} \equiv m_{i} \Sigma V$ is of order 1). A systematic expansion can be performed in which one is working to a given fixed order in $\epsilon$ [2].

In the above regime the leading term of the chiral Lagrangian is given by the zero momentum mode of the field $U(x)$. The method of ref. [2] treats this case by means of a collective field technique. To this aim, introduce a new variable $\xi(x)$ containing non-zero momentum modes only, and write

$$
U(x)=u e^{i \sqrt{2} \xi(x) / F} u,
$$


where $u \in \mathrm{U}\left(N_{f}+N_{v}\right)$ is a constant collective field corresponding to the zero momentum modes of the pseudo-Goldstone bosons. Let $U_{0} \equiv u^{2}$, and introduce the flavor singlet

$$
\Xi_{0} \equiv \operatorname{Tr} \varphi_{0}, \quad \text { with } \quad U_{0} \equiv \exp \left[i \sqrt{2} \varphi_{0} / F\right]
$$

and $\Xi(x) \equiv \operatorname{Tr} \xi(x)$. The change of variables (6) is next inserted into the path integral. The Jacobian is non-trivial, but to lowest order it contains a term that only shift the vacuum energy, without affecting the effective Lagrangian calculation below. We then find that the action to lowest order becomes

$$
\begin{aligned}
\int d x \mathcal{L}= & \int d x \operatorname{Tr}\left[\frac{1}{2} \partial_{\mu} \xi(x) \partial^{\mu} \xi(x)+\frac{m_{0}^{2}}{2 N_{c}}\left(\Xi(x)-\Xi_{0}\right)^{2}+\frac{\alpha}{2 N_{c}} \partial_{\mu} \Xi(x) \partial^{\mu} \Xi(x)\right] \\
& -\frac{\Sigma}{2} \operatorname{Tr}\left[\mathcal{M}\left(U_{0}+U_{0}^{\dagger}\right)\left(V-\frac{1}{F^{2}} \int d x \xi(x)^{2}\right)\right] .
\end{aligned}
$$

It is convenient to add to this action a mass term for the field $\xi(x)$, and only taking this mass to zero at the end of the calculations. Because of the Gaussian damping term for the non-zero modes, $\xi(x)$ counts as being of order $\epsilon$. For the singlet field $\Xi(x)$ the counting is not as straightforward. The kinetic energy term for $\Xi(x)$ suppresses fluctuations in $\Xi(x)$ beyond $\mathcal{O}(\epsilon)$, so that the mass term for $\Xi(x)$ is of order $\epsilon\left(m_{0}\right)^{2} \epsilon^{-2}$. The parameter $m_{0}$ is here a priori free as far as counting in $\epsilon$ is concerned, and we thus have exactly the same counting ambiguity as in conventional partially

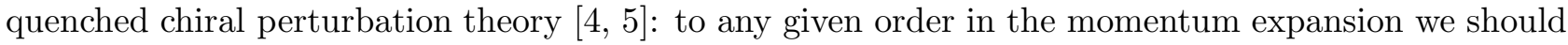
in principle include all orders of $m_{0}^{2}$ at each order in $M_{i j}^{2}$. What we need to know here is that we can take $m_{0}^{2} /\left(N_{c} F^{2}\right)$ to be parametrically small, and thus perform a simultaneous expansion in $\epsilon$ and $m_{0}^{2} /\left(N_{c} F^{2}\right)$. This is what we will do below.

Integrating out the fluctuation field $\xi(x)$ to one-loop order, we obtain the one-loop improved effective Lagrangian for the zero momentum modes. We are particularly interested in the first correction to the chiral condensate $\Sigma$. What enters repeatedly in what follows is the pseudo-Goldstone boson propagator, evaluated at zero,

$$
\Delta\left(M_{i j}^{2}\right) \equiv \frac{1}{V} \sum_{p} \frac{1}{p^{2}+M_{i j}^{2}}
$$

and the corresponding quantity for the modes without zero momentum,

$$
\bar{\Delta}\left(M_{i j}^{2}\right) \equiv \frac{1}{V} \sum_{p}{ }^{\prime} \frac{1}{p^{2}+M_{i j}^{2}} .
$$

The two are hence related by $\Delta\left(M_{i j}^{2}\right)=\left(M_{i j}^{2} V\right)^{-1}+\bar{\Delta}\left(M_{i j}^{2}\right)$.

It is instructive to first rederive the known finite-volume correction for the unquenched theory [2] in the present formulation. We thus evaluate the one-loop $\xi$-integral saturation of

$$
\left\langle\frac{m_{s} \Sigma}{2 F^{2}} \operatorname{Tr}\left[\left(U_{0}+U_{0}^{\dagger}\right) \int d x \xi(x)^{2}\right]\right\rangle_{\xi}
$$

where for convenience we have taken the $N_{f}$ dynamical fermions to be of equal mass $m_{s}$ (and the pseudo-Goldstone bosons thus have common masses $M_{s s}=2 m_{s} \Sigma / F^{2}$ ). Taking the $N_{v}=0$ expressions from above, we get ${ }^{1}$

$$
\left\langle\int d x \delta \mathcal{L}\right\rangle_{\xi}=\frac{V m_{s} \Sigma}{2 F^{2}} \operatorname{Tr}\left(U_{0}+U_{0}^{\dagger}\right)\left\{\left(N_{f}-1\right) \bar{\Delta}\left(M_{s s}^{2}\right)+\frac{1}{V} \sum_{p}{ }^{\prime} G\left(M_{s s}^{2}\right)\right\}
$$

\footnotetext{
${ }^{1}$ The coupling between $\Xi_{0}$ and $\Xi(x)$ is treated perturbatively. It contributes only to the next order.
} 


$$
=\frac{V m_{s} \Sigma}{2 F^{2}} \operatorname{Tr}\left(U_{0}+U_{0}^{\dagger}\right)\left\{\left(N_{f}-1\right) \bar{\Delta}\left(M_{s s}^{2}\right)+\bar{\Delta}\left(M_{s s}^{2}\right)-\frac{1}{V} \sum_{p}{ }^{\prime} \frac{\frac{1}{N_{c}}\left(m_{0}^{2}+\alpha p^{2}\right)}{\left(p^{2}+M_{s s}^{2}\right)^{2} \mathcal{F}\left(p^{2}\right)}\right\}(11)
$$

This differs from the expression quoted in ref. [2] because we have here included the effect of the flavor singlet field. Due to the anomaly, the mass of this field stays finite in the full theory as the quark masses are decreased, while the pseudo-Goldstone bosons approach zero mass. This corresponds to taking $m_{0}^{2} \rightarrow \infty$ in the above expression, which after setting the argument of the propagator to zero gives

$$
\left\langle\int d x \delta \mathcal{L}\right\rangle_{\xi}=\frac{V m_{s} \Sigma}{2 F^{2}} \operatorname{Tr}\left(U_{0}+U_{0}^{\dagger}\right)\left\{\frac{N_{f}^{2}-1}{N_{f}} \bar{\Delta}(0)\right\},
$$

and we hence recover the result of ref. [2].

We now turn to the fully quenched case. This simply corresponds to setting $N_{f}=0$ in the Feynman rules given above. We evaluate

$$
\lim _{N_{v} \rightarrow 0} \frac{1}{N_{v}}\left\langle\frac{m_{v} \Sigma}{2 F^{2}} \operatorname{Tr}\left[\left(U_{0}+U_{0}^{\dagger}\right) \int d x \xi(x)^{2}\right]\right\rangle
$$

which is a finite quantity. In complete analogy with eq. (11), this results in

$$
\begin{aligned}
& \lim _{N_{v} \rightarrow 0} \frac{1}{N_{v}} \frac{V m_{v} \Sigma}{2 F^{2}} \operatorname{Tr}\left(U_{0}+U_{0}^{\dagger}\right)\left\{\left(N_{v}-1\right) \bar{\Delta}\left(M_{v v}^{2}\right)+\frac{1}{V} \sum_{p}{ }^{\prime} G\left(M_{v v}^{2}\right)\right\}_{N_{v} \rightarrow 0} \\
& =\lim _{N_{v} \rightarrow 0} \frac{1}{N_{v}} \frac{V m_{v} \Sigma}{2 F^{2}} \operatorname{Tr}\left(U_{0}+U_{0}^{\dagger}\right)\left\{\left(N_{v}-1\right) \bar{\Delta}\left(M_{v v}^{2}\right)+\bar{\Delta}\left(M_{v v}^{2}\right)-\frac{1}{V} \sum_{p}{ }^{\prime} \frac{\frac{1}{N_{c}}\left(m_{0}^{2}+\alpha p^{2}\right)}{\left(p^{2}+M_{v v}^{2}\right)^{2} \mathcal{F}\left(p^{2}\right)}\right\}_{N_{v} \rightarrow 0} \\
& =-\lim _{N_{v} \rightarrow 0} \frac{1}{N_{v}} \frac{V m_{v} \Sigma}{2 F^{2}} \operatorname{Tr}\left(U_{0}+U_{0}^{\dagger}\right) \frac{1}{V} \sum_{p} \frac{\frac{1}{N_{c}}\left(m_{0}^{2}+\alpha p^{2}\right)}{\left(p^{2}+M_{v v}^{2}\right)^{2}},
\end{aligned}
$$

after doing the integration over the non-zero modes only. As expected, this involves the quenched double pole [4, 5]. We can rewrite the correction as

$$
-\lim _{N_{v} \rightarrow 0} \frac{1}{N_{v}} \frac{V m_{v} \Sigma}{2 N_{c} F^{2}} \operatorname{Tr}\left(U_{0}+U_{0}^{\dagger}\right)\left\{\alpha \bar{\Delta}\left(M_{v v}^{2}\right)-\left(m_{0}^{2}-\alpha M_{v v}^{2}\right) \partial_{M_{v v}^{2}} \bar{\Delta}\left(M_{v v}^{2}\right)\right\} .
$$

Let us summarize what this correction implies. To zero'th order, the mass-dependent quenched chiral condensate in finite volume $V$ is simply given by a group integral. We introduce the partition function at zero $\theta$-angle

$$
\mathcal{Z}\left(\mu_{v}\right)=\int_{U\left(N_{v}\right)} d U_{0} \exp \left[\frac{1}{2} \mu_{v} \operatorname{Tr}\left(U_{0}+U_{0}^{\dagger}\right)-\frac{m_{0}^{2} V}{2 N_{c}} \Xi_{0}^{2}\right] .
$$

It follows from the above analysis that this is the zero'th order approximation of the effective euclidean partition function of QCD with $N_{v}$ mass-degenerate (light) quarks in the finite-volume regime $V \gg$ $1 / \Lambda_{Q C D}^{4}$ and $M_{v v}^{4} \ll 1 / V$ [7], including the effects of a possibly light flavor singlet. Use has been made of the fact that the first term in the effective Lagrangian depends only on the combination $\mu_{v} \equiv m_{v} V \Sigma$. Formally the quenched chiral condensate then follows from

$$
\frac{\Sigma\left(\mu_{v}\right)}{\Sigma} \equiv \lim _{N_{v} \rightarrow 0} \frac{1}{N_{v}} \frac{\partial}{\partial \mu_{v}} \ln \mathcal{Z}
$$

While it may be difficult to evaluate this expression explicitly, both low-mass and large-mass series expansions have been obtained in sectors of fixed topological gauge field charge $\nu$ [8, [] 
To one-loop order in the fluctuations of the non-zero momentum modes we see that this result is modified in a simple way. One can use the same effective partition function (14) after replacing $\mu_{v}$ by

$$
\mu_{v}^{\prime} \equiv \mu_{v}\left\{1+\frac{1}{N_{c} F^{2}}\left[\alpha \bar{\Delta}\left(M_{v v}^{2}\right)-\left(m_{0}^{2}-\alpha M_{v v}^{2}\right) \partial_{M_{v v}^{2}} \bar{\Delta}\left(M_{v v}^{2}\right)\right]\right\} .
$$

To the order shown, the mass-dependent chiral condensate still follows from eq. (15), after making the above substitution in the partition function (14), viz.,

$$
\frac{\Sigma\left(\mu_{v}\right)}{\Sigma} \equiv \lim _{N_{v} \rightarrow 0} \frac{1}{N_{v}} \frac{\partial}{\partial \mu_{v}^{\prime}} \ln \mathcal{Z}\left(\mu_{v}^{\prime}\right) \cdot \frac{\mu_{v}^{\prime}}{\mu_{v}}
$$

This is completely analogous to the case of dynamical quarks [2], where instead one should replace the corresponding parameter $\mu_{s}$ by its modification due to (12).

Let us now compare this result with what one gets in ordinary quenched chiral perturbation theory, which is valid in the opposite finite-volume regime where $V \gg 1 / M_{v v}^{4}$. Then, to one-loop order 44, 10]

$$
\frac{\Sigma\left(m_{v}\right)}{\Sigma}=1+\frac{1}{N_{c} F^{2}}\left[\alpha \Delta\left(M_{v v}^{2}\right)-\left(m_{0}^{2}-\alpha M_{v v}^{2}\right) \partial_{M_{v v}^{2}} \Delta\left(M_{v v}^{2}\right)\right]+\ldots,
$$

which also immediately follows from the quenched Feynman rules given above. As in the case of dynamical fermions [2] there is an overlapping region where the two different expansions match on to each other. To see this, let us evaluate the leading-order contribution to the large- $\mu_{v}$ expansion based on the relations (14) and (15). We thus need the asymptotic expansion of the partition function (14) for $N_{v}$ mass-degenerate fermions. To the best of our knowledge, such an expansion has not been worked out yet. However, we can make use of the known asymptotic expansions in sectors of fixed topological gauge field charge $\nu$ [8, 9], and then perform the sum over topology subsequently [11]. We first note that the inclusion of an arbitrary vacuum angle $\theta$ in the effective partition function (14) can be re-absorbed into a shifted $\Xi_{0}$, so that

$$
\mathcal{Z}\left(\mu_{v}\right)=\int_{U\left(N_{v}\right)} d U_{0} \exp \left[\frac{1}{2} \mu_{v} \operatorname{Tr}\left(U_{0}+U_{0}^{\dagger}\right)-\frac{m_{0}^{2} V}{2 N_{c}}\left(\Xi_{0}-\theta F / \sqrt{2}\right)^{2}\right] .
$$

The projection on sectors of fixed topological charge $\nu$ is then performed by means of a Fourier transform [7]. The integral is Gaussian, and the result is, for large four-volumes $V$,

$$
\begin{aligned}
\mathcal{Z}_{\nu}\left(\mu_{v}\right) & =\frac{1}{2 \pi} \int_{0}^{2 \pi} d \theta e^{-i \nu \theta} \mathcal{Z}\left(\mu_{v}\right) \\
& =\frac{1}{\sqrt{\left\langle\nu^{2}\right\rangle}} e^{-\nu^{2} / 2\left\langle\nu^{2}\right\rangle} \int_{U\left(N_{v}\right)} d U_{0}\left(\operatorname{det} U_{0}\right)^{\nu} \exp \left[\frac{1}{2} \mu_{v} \operatorname{Tr}\left(U_{0}+U_{0}^{\dagger}\right)\right],
\end{aligned}
$$

up to an irrelevant $\nu$-independent normalization factor. We have then simultaneously identified the topological susceptibility [10]

$$
\left\langle\nu^{2}\right\rangle \equiv \frac{F^{2} m_{0}^{2} V}{2 N_{c}}
$$

We now insert the large- $\mu_{v}$ series expansion of $\mathcal{Z}_{\nu}\left(\mu_{v}\right)$ (up to $\mu_{v}$-independent factors that are irrelevant right here) [8, 9],

$$
\mathcal{Z}_{\nu}\left(\mu_{v}\right)=\mu_{v}^{-N_{v}^{2} / 2} e^{N_{v} \mu_{v}}\left(1-N_{v} \frac{\nu^{2}}{2 \mu_{v}}+N_{v}\left(2 N_{v}^{2}+1\right) \frac{1}{8 \mu_{v}}+\ldots\right)
$$


into the definition of the quenched chiral condensate

$$
\lim _{N_{v} \rightarrow 0} \frac{1}{N_{v}} \frac{\partial}{\partial \mu_{v}} \ln \mathcal{Z}\left(\mu_{v}\right)=1+\frac{\nu^{2}}{2 \mu_{v}^{2}}-\frac{1}{8 \mu_{v}^{2}}+\ldots=1+\frac{\nu^{2}-1 / 4}{2 \mu_{v}^{2}}+\ldots
$$

When next we wish to sum over topology [1],

$$
\frac{\Sigma\left(\mu_{v}\right)}{\Sigma}=\frac{\left\langle\Sigma_{\nu}\left(\mu_{v}\right)\right\rangle}{\Sigma}=\frac{1}{\Sigma} \sum_{\nu=-\infty}^{\infty} \mathcal{Z}_{\nu}\left(\mu_{v}\right) \Sigma_{\nu}\left(\mu_{v}\right) / \mathcal{Z}\left(\mu_{v}\right)
$$

we are faced with the fact that $\left\langle\nu^{2}\right\rangle \sim V$, while $\mu_{v}$ here is kept finite as $V \rightarrow \infty$. This means that it is not immediately obvious if there is a region in which we can expect to find overlap between the two perturbative expansions discussed above.2 The precise requirement for the $\nu$-average of the above first-order term in the expansion to be small is

$$
V \gg \frac{F^{2} m_{0}^{2}}{2 N_{c} m_{v}^{2} \Sigma^{2}}
$$

while we also need

$$
V \ll \frac{1}{M_{v v}^{4}}=\frac{F^{4}}{4 m_{v}^{2} \Sigma^{2}} .
$$

This can be achieved by having $m_{0}^{2} / N_{c} \ll F^{2} / 2$, which formally can be met by sending $N_{c} \rightarrow \infty$ while keeping all other quantities fixed[1. As discussed above, this limit is within the region of validity of the finite-volume $\epsilon$-expansion, and it gives a consistent regime in which we should have simultaneous validity of both types of expansions. We can then insert the relation (21) into the $\nu$-averaged condensate

$$
\lim _{N_{v} \rightarrow 0}\left\langle\frac{1}{N_{v}} \frac{\partial}{\partial \mu_{v}} \ln \mathcal{Z}_{\nu}\left(\mu_{v}\right)\right\rangle \simeq 1+\frac{\left\langle\nu^{2}\right\rangle}{2 \mu_{v}^{2}}+\ldots=1+\frac{m_{0}^{2}}{N_{c} F^{2} M^{4} V}+\ldots,
$$

and hence, from eqs. (17) and (16), to leading order

$$
\begin{aligned}
\frac{\Sigma\left(\mu_{v}\right)}{\Sigma} & =\left[1+\frac{m_{0}^{2}}{N_{c} F^{2} M^{4} V}\right]\left\{1+\frac{1}{N_{c} F^{2}}\left[\alpha \bar{\Delta}\left(M_{v v}^{2}\right)-\left(m_{0}^{2}-\alpha M_{v v}^{2}\right) \partial_{M_{v v}^{2}} \bar{\Delta}\left(M_{v v}^{2}\right)\right]\right\}+\ldots \\
& =1+\frac{1}{N_{c} F^{2}}\left[\alpha \Delta\left(M_{v v}^{2}\right)-\left(m_{0}^{2}-\alpha M_{v v}^{2}\right) \partial_{M_{v v}^{2}} \Delta\left(M_{v v}^{2}\right)\right]+\ldots
\end{aligned}
$$

Thus, in this particular regime the leading-order correction to the chiral condensate precisely coincides with that of one-loop chiral perturbation theory (18).

An alternative way of interpreting the formulas (16) and (17) is that, effectively, and to leading order in the finite-volume perturbation theory, one is working with a shifted quenched chiral condensate at finite volume:

$$
\Sigma_{e f f}=\Sigma\left\{1+\frac{1}{N_{c} F^{2}}\left[\alpha \bar{\Delta}\left(M_{v v}^{2}\right)-\left(m_{0}^{2}-\alpha M_{v v}^{2}\right) \partial_{M_{v v}^{2}} \bar{\Delta}\left(M_{v v}^{2}\right)\right]\right\} .
$$

\footnotetext{
${ }^{2}$ Indeed, the leading-order term in the expansion, which ordinarily is responsible for an overlapping region of validity [2, 10 of the two expansions is proportional to $N_{v}$, and hence vanishes in the quenched case. As shown below, in the quenched theory the region of agreement is just pushed one order higher.

${ }^{3}$ In a proper scaling with $N_{c}, m_{0}$ is of order 1 , while $F^{2}$ is of order $N_{c}$, but this only makes it more clear that the inequality can be met.
} 
It is clearly of interest to have this volume dependence made more explicit. So far the calculation has been made at fixed ultraviolet cut-off $\Lambda_{U V}$ (having in mind a lattice cut-off with scale $\Lambda_{U V} \sim 1 / a$ ). The explicit volume dependence is recovered once one notices that in most regularization schemes the boson propagator at the origin (10), and any derivative thereof, separates into ultraviolet divergent volumeindependent terms, and additional finite volume-dependent terms [12]. The theory is renormalized as at infinite volume, and the explicit volume dependence is what remains. A detailed analysis has been made by Hasenfratz and Leutwyler [12], from where we can borrow the main results. In dimensional regularization, and setting the subtraction point equal to unity, one has

$$
\Delta\left(M^{2}\right)=\frac{M^{2}}{16 \pi^{2}}\left[\ln \left(M^{2}\right)+c_{1}\right]+g_{1}\left(M^{2}, L_{i}\right)
$$

where $c_{1}$ is a mass and $L_{i}$ independent constant that diverges near four dimensions. We have ignored the tree level contribution from the $\mathcal{O}\left(p^{4}\right)$ terms in the chiral Lagrangian; they give volume-independent terms that are of no interest here. All volume dependence is contained in the functions (in four dimensions; for $\Delta\left(M^{2}\right)$ we need only $r=1$ ) [2, 12]

$$
g_{r}\left(M^{2}, L_{i}\right) \equiv \frac{1}{V}\left(\frac{L^{2}}{4 \pi}\right)^{r} \int_{0}^{\infty} d t t^{r-3} \exp \left[-\frac{M^{2} L^{2} t}{4 \pi}\right]\left(\prod_{i=1}^{4} S\left(L_{i}^{2} /(t L)\right)-1\right)
$$

where

$$
S(x)=\sum_{n=-\infty}^{\infty} \exp \left[-\pi n^{2} x\right]
$$

The general relation

$$
g_{r}\left(M^{2}, L_{i}\right)=-\frac{\partial}{\partial M^{2}} g_{r-1}\left(M^{2}, L_{i}\right)
$$

allows one to calculate all $g_{r}$ 's from $g_{0}\left(M^{2}, L_{i}\right)$. For small masses a systematic expansion has been given in ref. [12]:

$$
g_{0}\left(M^{2}, L_{i}\right)=-\frac{2}{V} \ln (M L)+\frac{1}{16 \pi^{2}} M^{4}\left[\ln (M L)-\frac{1}{4}\right]+\frac{1}{V} \sum_{n=0}^{\infty} \frac{1}{n !} \beta_{n}\left(L_{i} / L\right)(M L)^{2 n}
$$

where $\beta_{n}\left(L_{i} / L\right)$ are "shape coefficients" that depend on the shape of the four-volume, and which can easily be evaluated numerically using the formulas of ref. [12]. Subtracting the zero-momentum pole term, we thus have

$$
\begin{aligned}
\bar{g}_{1}\left(M^{2}, L_{i}\right) & =g_{1}\left(M^{2}, L_{i}\right)-\frac{1}{M^{2} V} \\
& =\frac{1}{8 \pi^{2}} M^{2} \ln (M L)-\sum_{n=1}^{\infty} \frac{1}{(n-1) !} \beta_{n}\left(L_{i} / L\right) M^{2(n-1)} L^{2 n-4}
\end{aligned}
$$

and

$$
\bar{g}_{2}\left(M^{2}, L_{i}\right)=\frac{1}{8 \pi^{2}} \ln (M L)+\frac{1}{16 \pi^{2}}+\sum_{n=2}^{\infty} \frac{1}{(n-2) !} \beta_{n}\left(L_{i} / L\right) M^{2(n-2)} L^{2 n-4},
$$

from which we obtain both

$$
\bar{\Delta}\left(M^{2}\right)=\frac{M^{2}}{8 \pi^{2}}\left(c_{1}-\ln (L)\right)-\sum_{n=1}^{\infty} \frac{1}{(n-1) !} \beta_{n}\left(L_{i} / L\right) M^{2(n-1)} L^{2 n-4}
$$


and its derivative in a small-mass expansion. All volume dependence of the one-loop result (29) is thus explicitly given.

We note two important differences between the one-loop correction to the quenched chiral condensate, and that of the full theory with dynamical quarks:

- The expression for the effective finite-volume chiral condensate (29) involves two new constants $\left(m_{0}\right.$ and $\left.\alpha\right)$, in addition to the pion decay constant $F$.

- In contrast to the case of dynamical quarks (12), the leading correction contains not just the finite volume function $g_{1}\left(M_{v v}^{2}, L_{i}\right)$, but also $g_{2}\left(M_{v v}^{2}, L_{i}\right)$.

The first point means that it is not straightforward to use the quenched chiral condensate to extract the (quenched) pion decay constant $F$. The second point implies, as we will see shortly, that the finite-volume perturbative expansion of this quantity contains a logarithmic divergence.

We wish to find the effective chiral condensate at the finite volumes considered here. To avoid specifying the scale entering the logarithm and all volume-independent terms, it is convenient to simply compare $\Sigma_{\text {eff }}$ at two different four-volumes $V_{1}$ and $V_{2}$ of same geometry (here, let $L_{1,2} \equiv V_{1,2}^{1 / 4}$ ). Based on (29) and (38), we find

$$
\frac{\Sigma_{e f f}\left(V_{1}\right)}{\Sigma_{e f f}\left(V_{2}\right)}=1-\frac{1}{N_{c} F^{2}}\left[\alpha \beta_{1}\left(\frac{1}{L_{1}^{2}}-\frac{1}{L_{2}^{2}}\right)-\frac{m_{0}^{2}}{8 \pi^{2}} \ln \left(L_{1} / L_{2}\right)\right]+\ldots
$$

after having taken the limit $M_{v v} \rightarrow 0$. In contrast to the case of dynamical quarks, what should be a small finite-volume correction can in fact become arbitrarily large. This logarithmic divergence of the effective chiral condensate as the volume $V$ is sent to infinity is a direct analog of the well-known

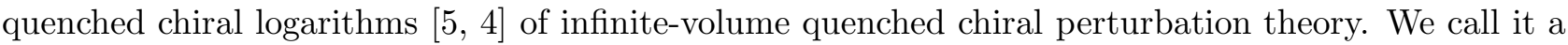
quenched finite volume logarithm.

A logarithmic divergence in what should be a small correction of course signals that this finite-volume perturbative expansion of the quenched effective theory is problematic. The trouble that shows up as $\ln \left(M_{v v}\right)$ for $M_{v v} \rightarrow 0$ in the regime $L \gg 1 / M_{v v}$, shows up as $\ln (L)$ in the finite-volume regime where $L \ll 1 / M_{v v}$. Roughly speaking, as $1 / M_{v v}$ is lowered and crosses $L$, the infrared cut-off switches from being given by $1 / M_{v v}$ to being given by $L$. For both expansions it is the very starting point of the expansion that appears internally inconsistent. It seems that at best one can hope that either of the two perturbative expansions have windows of validity where the one-loop corrections remain small. If we adopt this point of view, then we should clearly demand in our case that when comparing $\Sigma_{\text {eff }}$ at two different volumes $V_{1}$ and $V_{2}$ we must require

$$
\frac{m_{0}^{2}}{8 \pi^{2} N_{c} F^{2}}\left|\ln \left(L_{1} / L_{2}\right)\right| \ll 1
$$

in order to apply (39). It has been argued by Sharpe [5] that in usual chiral perturbation theory it may be possible to resum a certain class of diagrams that give rise to quenched chiral logarithms. However, also this partial resummation remains divergent. Presumably a similar resummation may be performed for the quenched finite volume logarithms. But we prefer to avoid speculation beyond the one-loop order to which we have computed the correction, and insist at least on the condition (40).

\footnotetext{
${ }^{4}$ The function $g_{2}\left(M_{s s}^{2}, L_{i}\right)$ appears at next order in the unquenched case, but then it is suppressed by an additional factor of the volume $V$, and hence harmless.
} 
Difficulties with quenched chiral perturbation theory in finite volumes (but associated with unusual power-law behavior in $L$ ) have been noted earlier in connection with the quenched pion scattering length [13].

A divergent chiral condensate for the quenched theory has long been suspected from many different arguments, of which the presence of ordinary (infinite-volume) quenched chiral logarithms 河, 何 is the one closest to the present perspective. The divergence has been difficult to see in lattice gauge theory simulations, but a recent careful Monte Carlo study of the quenched Schwinger model using overlap fermions has revealed a clear increase in $\Sigma$ as the volume $V$ is made larger 14. This should be directly related to the quenched finite volume logarithm found here, although the analysis will differ in detail due to the particularities of two space-time dimensions [15, 16]

\section{The Chiral Condensate and $F$ from Dirac Operator Eigenvalues}

The dominance of the zero momentum mode in the effective chiral Lagrangian when $1 / M_{i i}^{4} \gg V$ is known to have a very direct consequence in terms the Dirac operator spectrum of the underlying theory 17, 17, 18. In this finite-volume range the spectral properties of the Dirac operator are analytically computable for an infinite sequence of eigenvalues. While originally derived on the basis of universal results from Random Matrix Theory [17, 18], the low-energy spectrum of the Dirac operator can equally well be obtained directly from the effective Lagrangian framework [10, 19, 20, 21]. Let us denote the spectral density of the Dirac operator by $\rho\left(\lambda ; m_{1}, \ldots, m_{N_{f}}\right)$, where $\lambda$ is the eigenvalue. It was observed some time ago [22] that the so-called microscopic spectral density [17],

$$
\rho_{s}\left(\zeta ; \mu_{1}, \ldots, \mu_{N_{f}}\right) \equiv \frac{1}{V \Sigma} \rho\left(\frac{\zeta}{V \sigma} ; \frac{\mu_{1}}{V \Sigma}, \ldots, \frac{\mu_{N_{f}}}{V \Sigma}\right)
$$

gives an excellent finite-size scaling function from which to extract the infinite-volume chiral condensate $\Sigma$ from finite lattice volumes. Even more information can be obtained from the individual eigenvalue distributions, which all depend on just this one single parameter $\Sigma$, and whose complete analytical expressions are now known [23]. Each single eigenvalue distribution can then be used to measure the infinite-volume chiral condensate $\Sigma$.

The preceding section clearly suggests that apart from the chiral condensate one more quantity can be extracted from the microscopic Dirac operator spectrum, namely the pion decay constant $F$. While the quenched case is special because of the quenched finite volume logarithm, the situation is much more favorable when considering a theory with dynamical quarks. We recall that the effective partition function, including its one-loop correction (12), can be written

$$
\mathcal{Z}_{e f f}=\int_{S U\left(N_{f}\right)} d U \exp \left[\frac{1}{2} m \Sigma_{e f f} V \operatorname{Tr}\left(U_{0}+U_{0}^{\dagger}\right)\right] .
$$

where

$$
\Sigma_{e f f}=\Sigma\left[1-\frac{N_{f}^{2}-1}{N_{f}} \frac{1}{F^{2}} \bar{\Delta}\left(M_{s s}^{2}\right)\right] .
$$

Taking the massless limit of eq. (43), the only correction to $\Sigma$ comes from the finite four-volume:

$$
\Sigma_{e f f}=\Sigma\left[1+\frac{N_{f}^{2}-1}{N_{f}} \frac{1}{F^{2}} \frac{\beta_{1}\left(L_{i} / L\right)}{L^{2}}+\mathcal{O}\left(1 / L^{4}\right)\right] .
$$


For symmetric four-tori $\beta_{1}=0.140461 \ldots$ [12], and for other shapes the coefficient $\beta_{1}$ is readily computed numerically from the general formula of that same reference. The correction to $\Sigma$ is thus explicitly known to one-loop accuracy, and it depends only on $F$.

From the effective partition function (42), and its supersymmetric extension [10, 19, 20], follows the microscopic Dirac operator spectrum. To first order in perturbation theory it is unchanged in form compared with the leading behavior, except for the finite-volume shift indicated in (43). It is immediately clear from (42) that all predictions for fixed sectors of topological charge $\nu$ are unchanged as well, except for the shift in $\Sigma$. With high enough statistics, the finite volume correction to $\Sigma$ can be measured from fits to different lattice volumes, yielding $F$. The two-loop contribution to the chiral condensate in this volume range has also been computed [24], but at that order one can no longer view the correction as just a shift in $\Sigma$ in an otherwise unaffected effective theory. The suggestion that the pion decay constant can be extracted using finite-volume scaling in this form was first made by Gasser and Leutwyler [2], and in a slightly different setting by Neuberger [25]. The Dirac operator eigenvalues just give a particularly convenient way to make use of the formalism.

The possibility of measuring the pion decay constant $F$ by considering the combination of chiral perturbation theory and the analytical results for the microscopic Dirac operator spectrum was first explored by Berbenni-Bitsch et al. in ref. [26]. They compared lattice Monte Carlo data for the chiral condensate in quenched $\mathrm{SU}(2)$ gauge theory with a general expansion of $\Sigma(m)$ for staggered fermions at finite four-volumes. Relating one of the coefficients in the expansion to the pion decay constant $F$ by means of the Gell-Mann-Oakes-Renner relation, they were thus able to extract $F$ from a general fit of $\Sigma(m)$ over a mass range of several orders of magnitude. A similar analysis has very recently been made for staggered fermions and gauge group $\mathrm{SU}(3)$ [27]. In that case there are independent estimates of $F$ from earlier lattice studies, and the authors of ref. [27] find very good agreement.

A comment on staggered fermions can be made here. Comparisons between the analytical prediction for the Dirac operator spectrum of dynamical staggered fermions will typically be made so far from the continuum that the continuum number of flavors is not yet seen. Instead, the theory appears for just one staggered fermion to be of $N_{f}=1$ [28], and in the effective theory the remaining bosons are heavy on the scale of the one single pseudo-Goldstone boson. The symmetry breaking pattern is thus $\mathrm{U}(1) \times \mathrm{U}(1) \rightarrow \mathrm{U}(1)$, and it is of interest to know also the leading correction for an unquenched theory with this Abelian Goldstone manifold. Doing the one-loop finite-volume perturbative calculation we immediately find the analog of eq. (12):

$$
\left\langle\int d x \delta \mathcal{L}\right\rangle_{\xi}=\frac{V m_{s} \Sigma}{2 F^{2}}\left(U_{0}+U_{0}^{\dagger}\right) \bar{\Delta}\left(M_{s s}^{2}\right)
$$

where in this case $U_{0} \in \mathrm{U}(1)$. The effective chiral condensate is then rescaled according to

$$
\Sigma_{e f f}=\Sigma\left[1-\frac{1}{F^{2}} \bar{\Delta}(0)\right]
$$

Thus also in this $N_{f}=1$ theory one can extract the pion decay constant $F$ from the smallest Dirac operator eigenvalue distributions. The general case of $N_{f}$ staggered fermions at strong coupling follows similarly (a factor of $N_{f}$ in front of the correction term in (46)).

The above result for the $N_{f}=1$ theory with staggered fermions away from the continuum limit may appear to contradict a recent lattice Monte Carlo study of the distributions of the smallest Dirac operator eigenvalues in $\mathrm{SU}(3)$ lattice gauge theory with one staggered fermion [28]. There a very 
accurate agreement was found when comparing with the analytical prediction based on the continuum $N_{f}=1$ theory, where there is no Goldstone manifold at all, and all mesons are massive even in the chiral limit. In fact, there is no contradiction. The agreement found in [28] was with the sector of just topological gauge field charge $\nu=0$. The projection on this sector is actually equivalent to having a U(1) Goldstone manifold, as needed in order to compare with the $N_{f}=1$ theory with staggered fermions away from the continuum. In detail, the projection on the $\nu=0$ sector,

$$
\mathcal{Z}_{\nu=0}=\frac{1}{2 \pi} \int_{0}^{2 \pi} d \theta \mathcal{Z}(\theta)=\int_{U(1)} d U e^{\mu \cos (\theta)}
$$

is completely equivalent to the zero momentum mode effective partition function for the coset of $\mathrm{U}(1) \times \mathrm{U}(1) \rightarrow \mathrm{U}(1)$ spontaneous symmetry breaking, without any projection on the $\nu=0$ sector. The rôle of the zero-mode $\mathrm{U}(1)$ field is simply being played by the angular integration that projects down on $\nu=0$. This also gives a complementary viewpoint to the observation that staggered fermions seem insensitive to gauge field topology at these couplings [29] (see ref. [30] for the picture that emerges as the continuum limit is taken).

\section{Conclusions}

We have analyzed quenched chiral perturbation theory in the finite-volume regime where the pseudoGoldstone masses have Compton wavelengths much larger than the linear extent of the volume, $1 / M_{s s}^{4} \gg V$. In this regime a good starting point for perturbation theory is not the usual planewave solution of the fields. Rather, it is a collective variable which is chosen to be the zero momentum mode of the given field. Using this perturbation theory, and adding the (uniterated) vertex corresponding to the singlet field $\Phi_{0}(x)$ as usual for the quenched theory, we have computed the one-loop correction to the quenched chiral condensate. It turns out that this correction becomes large when the volume is taken to infinity, in the chiral limit as a logarithm in the volume $V$. This so-called quenched finite volume logarithm prohibits taking the volume $V$ to infinity. Whether or not it signals a true divergence in the quenched chiral condensate clearly cannot be ascertained within this perturbative framework alone. Quenched finite volume logarithms are likely to be a generic feature of quenched chiral perturbation theory in the present finite-volume regime, and should thus appear in other observables as well.

In the full theory with dynamical quarks the one-loop correction to the chiral condensate in this finite-volume regime amounts to a rescaling of $\Sigma$ in an otherwise unchanged effective theory. We have used this fact to point out that the microscopic spectrum of the Dirac operator, and in particular the individual smallest eigenvalue distributions, can be used to extract the pion decay constant $F$. This entails determining numerically the leading correction to the well-known microscopic scaling for the Dirac operator spectrum near the origin, and it appears to be feasible. It is quite different from the program to extract these quantities and further couplings in the chiral Lagrangian from (ratios of) matrix elements whose expansions in partially quenched chiral perturbation theory are known [31, 32]. So far the eigenvalue approach has only been tested away from the continuum and only for the case of $\Sigma$. It would be interesting to see if with present lattice volumes the finite-volume corrections can be determined to the accuracy required to extract $F$, and can be pursued towards the continuum.

\section{Acknowledgments}

This work was supported in part by EU TMR grant no. ERBFMRXCT97-0122. The author thanks J. Bijnens, C. Diamantini and P. Hernandez for discussions. 


\section{References}

[1] J. Gasser and H. Leutwyler, Phys. Lett. B 184 (1987) 83.

[2] J. Gasser and H. Leutwyler, Phys. Lett. B 188 (1987) 477.

[3] P. H. Damgaard and K. Splittorff, Phys. Rev. D 62 (2000) 054509 hep-lat/0003017.

[4] C. W. Bernard and M. F. Golterman, Phys. Rev. D 46 (1992) 853 hep-lat/9204007; Phys. Rev. D 49 (1994) 486 hep-lat/9306005.

M. F. Golterman and K. Leung, Phys. Rev. D 57 (1998) 5703 hep-lat/9711033.

[5] S. R. Sharpe, Phys. Rev. D 41 (1990) 3233; Phys. Rev. D 46 (1992) 3146 hep-lat/9205020; Phys. Rev. D 56 (1997) 7052 [Erratum-ibid. D 62 (1997) 7052] [hep-lat/9707018].

[6] G. Colangelo and E. Pallante, Nucl. Phys. B 520 (1998) 433 hep-lat/9708005.

[7] H. Leutwyler and A. Smilga, Phys. Rev. D 46 (1992) 5607.

[8] P. H. Damgaard and K. Splittorff, Nucl. Phys. B 572 (2000) 478 [hep-th/9912146].

P. H. Damgaard, Phys. Lett. B 476 (2000) 465 hep-lat/0001002].

[9] D. Dalmazi and J. J. Verbaarschot, Nucl. Phys. B 592 (2001) 419 hep-th/0005229.

[10] J. C. Osborn, D. Toublan and J. J. Verbaarschot, Nucl. Phys. B 540 (1999) 317 [hep-th/9806110].

[11] P. H. Damgaard, Nucl. Phys. B 556 (1999) 327 [hep-th/9903096.

[12] P. Hasenfratz and H. Leutwyler, Nucl. Phys. B 343 (1990) 241.

[13] C. W. Bernard and M. F. Golterman, Phys. Rev. D 53 (1996) 476 hep-lat/9507004.

[14] J. Kiskis and R. Narayanan, Phys. Rev. D 62 (2000) 054501 hep-lat/0001026.

[15] A. V. Smilga, Phys. Rev. D 46 (1992) 5598.

[16] S. Durr and S. R. Sharpe, Phys. Rev. D 62 (2000) 034506 hep-lat/9902007.

[17] E. V. Shuryak and J. J. Verbaarschot, Nucl. Phys. A 560 (1993) 306 [hep-th/9212088].

J. J. Verbaarschot and I. Zahed, Phys. Rev. Lett. 70 (1993) 3852 hep-th/9303012.

J. Verbaarschot, Phys. Rev. Lett. 72 (1994) 2531 hep-th/9401059.

[18] G. Akemann, P. H. Damgaard, U. Magnea and S. Nishigaki, Nucl. Phys. B 487 (1997) 721 hep-th/9609174.

[19] P. H. Damgaard, J. C. Osborn, D. Toublan and J. J. Verbaarschot, Nucl. Phys. B 547 (1999) 305 hep-th/9811212].

[20] D. Toublan and J. J. Verbaarschot, Nucl. Phys. B 560 (1999) 259 hep-th/9904199; hepth/0012144.

[21] R. J. Szabo, Nucl. Phys. B 598 (2001) 309 hep-th/0009237.

G. Akemann, D. Dalmazi, P. H. Damgaard and J. J. Verbaarschot, hep-th/0011072.

[22] M. E. Berbenni-Bitsch, A. D. Jackson, S. Meyer, A. Schafer, J. J. Verbaarschot and T. Wettig, Nucl. Phys. Proc. Suppl. 63 (1998) 820 hep-lat/9709102. 
[23] S. M. Nishigaki, P. H. Damgaard and T. Wettig, Phys. Rev. D 58 (1998) 087704 [hep-th/9803007]. P. H. Damgaard and S. M. Nishigaki, Phys. Rev. D 63 (2001) 045012 hep-th/0006111.

[24] F. C. Hansen, Nucl. Phys. B 345 (1990) 685.

[25] H. Neuberger, Phys. Rev. Lett. 60 (1988) 889; Nucl. Phys. B 300 (1988) 180.

[26] M. E. Berbenni-Bitsch, M. Gockeler, H. Hehl, S. Meyer, P. E. Rakow, A. Schafer and T. Wettig, Phys. Lett. B 466 (1999) 293 hep-lat/9907014.

[27] M. Gockeler, H. Hehl, P. E. Rakow, A. Schafer and T. Wettig, hep-lat/0105011.

[28] P. H. Damgaard, U. M. Heller, R. Niclasen and K. Rummukainen, Phys. Lett. B 495 (2000) 263 hep-lat/0007041.

[29] P. H. Damgaard, U. M. Heller, R. Niclasen and K. Rummukainen, Phys. Rev. D 61 (2000) 014501 hep-lat/9907019.

[30] F. Farchioni, I. Hip and C. B. Lang, Phys. Lett. B 471 (1999) 58 hep-lat/9909132.

[31] S. Sharpe and N. Shoresh, Phys. Rev. D 62 (2000) 094503 hep-lat/0006017.

[32] J. Heitger, R. Sommer and H. Wittig [ALPHA Collaboration], Nucl. Phys. B 588 (2000) 377 hep-lat/0006026. 